Letter to the Editor

Nicola Vanacore*, MD, PhD

\title{
New clinical research urgently needed for adjunctive OMT treatment in elderly patients hospitalized with COVID-19 pneumonia
}

https://doi.org/10.1515/jom-2020-0315

Received December 14, 2020; accepted December 24, 2020; published online February 18, 2021

To the Editor:

As of December 13, 2020, a total of 70,228,447 confirmed cases of coronavirus disease 2019 (COVID-19) - including 1,595,187 deaths - have been reported worldwide [1]. In a previous systematic review performed to demonstrate the clinical course and outcomes of COVID-19 pneumonia in critically ill patients, a $32.3 \%$ (21,145 of 65,383$)$ intensive care unit mortality rate was reported [2].

During the influenza epidemic of 1918, the American Osteopathic Association (AOA) distributed a survey to osteopathic physicians, querying them on mortality from influenza and pneumonia during that time [3]. The 2,445 physician respondents reported 110,122 cases of influenza and 257 fatalities, for a mortality rate of $0.23 \%$ compared with a national mortality rate of $12-15 \%$ [3]. The AOA survey results [3] also showed 6,258 case reports of influenza complicated by pneumonia, a rate of $10 \%$ [3] compared with the national average of $25 \%$ [4]. With those results in mind and in the setting of the current COVID-19 pandemic, I believe there is an urgent need to conduct clinical research to assess whether adjunctive OMT treatment can be useful in the management of elderly patients hospitalized with COVID-19 pneumonia.

From 1999 to 2016, an American-based osteopathic research group led by Noll published four randomized controlled trials (RCT) on adjunctive osteopathic manipulative treatment (OMT) in elderly patients hospitalized with pneumonia [5-8]. The first two RCTs included 21 [5] and 58 patients [6], respectively, who were at least 60 years old and hospitalized with acute

*Corresponding author: Nicola Vanacore, MD, PhD, National Center for Disease Prevention and Health Promotion, Italian National Institute of Health, Via Giano della Bella, 34, 00162, Rome, Italy, E-mail: nicola.vanacore@iss.it pneumonia. These RCTs [5, 6] - both of which should be considered pilot studies - demonstrated that patients assigned to the OMT group showed some clinical benefit compared with a control group.

Noll et al. [7] later performed a double-blind RCT in which 406 hospitalized subjects aged 50 years or older with pneumonia were randomly assigned to one of three groups: conventional care only (CCO), light-touch treatment (LT), or OMT. The protocol developed for that study [7] was in done in accordance with current clinical OMT applications [9]. An intention-to-treat (ITT) analysis found no differences between the three groups, while per protocol (PP) analysis found significant reductions in hospital length of stay, duration of intravenous antibiotics, respiratory failure, death, or discharge when the OMT group's results were compared with patients who received CCO [7]. In another subgroup ITT analysis [8] of adults aged 75 years or older, in-hospital mortality rates were lower for the OMT (1 of $66[2.0 \%]$ ) and LT (2 of 68 [3.0\%]) groups than the CCO group ( 9 of 67 [13.0\%]; $\mathrm{p}=0.005$ ). Specific clinical epidemiological parameters are reported in the Table [8].

Table: Measures of the effect of adjunctive OMT on in-hospital mortality among adults aged 75 years or older hospitalized for COVID-19 pneumonia [8] ${ }^{\mathrm{a}}$.

\begin{tabular}{|c|c|c|}
\hline Measure & Formula & Value \\
\hline $\begin{array}{l}\text { Mortality in the } \\
\text { control group (Mc) }\end{array}$ & $\begin{array}{l}M c=(n \text { deaths } / n \\
\text { participants }) \times 100\end{array}$ & $13.0 \%$ \\
\hline $\begin{array}{l}\text { Mortality in the } \\
\text { treatment group (Mt) }\end{array}$ & $\begin{array}{l}\mathrm{Mt}=(\mathrm{n} \text { deaths } / \mathrm{n} \\
\text { participants }) \times 100\end{array}$ & $2.0 \%$ \\
\hline $\begin{array}{l}\text { Absolute risk } \\
\text { reduction (ARR) }\end{array}$ & $A R R=M t-M c$ & $-11.0 \%$ \\
\hline Relative risk (RR) & $\mathrm{RR}=\mathrm{Mt} / \mathrm{Mc}$ & 0.15 \\
\hline $\begin{array}{l}\text { Relative risk } \\
\text { reduction (RRR) }\end{array}$ & $R R R=[(M c-M t) / M c] \times 100$ & $85.0 \%$ \\
\hline $\begin{array}{l}\text { Number needed to treat } \\
\text { (NNT) }\end{array}$ & $N N T=1 / A R R$ & 9 \\
\hline
\end{tabular}

${ }^{\mathrm{a}}$ Table developed by the authors from data published in Noll DR, Degenhardt BF, Johnson JC. Multicenter osteopathic pneumonia study in the elderly: subgroup analysis on hospital length of stay, ventilatordependent respiratory failure rate, and in-hospital mortality rate [8]. 
PP or subgroup analyses are considered exploratory analyses when conducting an RCT. Overall, the results of the 2016 Noll et al. [8] study were inconclusive, as exploratory analyses cannot be considered definitive and additional studies are needed in order to draw more definite conclusions. Unfortunately, there were no current studies on OMT in patients with COVID-19 pneumonia registered in the ClinicalTrials.gov and EudraCT databases as of December 14, 2020. A literature review was recently published that speculated about the potential use of OMT in COVID-19 patients, but additional rigorous clinical trials are required [10].

Again, with these limited studies in mind and in the midst of the current pandemic, there is an urgent need to generate clinical research about the ways in which OMT research might be useful in the management of elderly patients hospitalized with pneumonia complications from COVID-19.

Research funding: None declared.

Author contributions: The author has accepted responsibility for the entire content of this manuscript and approved its submission.

Competing interests: Author states no conflict of interest.

\section{References}

1. WHO coronavirus disease (COVID-19) dashboard. Available from: https://covid19.who.int [Accessed 13 Dec 2020].
2. Serafim RB, Póvoa P, Souza-Dantas V, Kalil AC, Salluh JIF. Clinical course and outcomes of critically ill patients with COVID-19 infection: a systematic review. Clin Microbiol Infect 2020. https:// doi.org/10.1016/j.cmi.2020.10.017.

3. Osteopathy's epidemic record. Osteopath Physician 1919;36:1.

4. Gevitz N. The DOs: osteopathic medicine in America. Baltimore, MD: The John Hopkins University Press; 1982:71-2 pp.

5. Noll DR, Shores J, Bryman PN, Masterson EV. Adjunctive osteopathic manipulative treatment in the elderly hospitalized with pneumonia: a pilot study. J Am Osteopath Assoc 1999;99: 143-52.

6. Noll DR, Shores JH, Gamber RG, Herron KM, Swift J Jr. Benefits of osteopathic manipulative treatment for hospitalized elderly patients with pneumonia. J Am Osteopath Assoc 2000;100: 776-82.

7. Noll DR, Degenhardt BF, Morley TF, Blais FX, Hortos KA, Hensel K, et al. Efficacy of osteopathic manipulation as an adjunctive treatment for hospitalized patients with pneumonia: a randomized controlled trial. Osteopath Med Prim Care 2010;4:2.

8. Noll DR, Degenhardt BF, Johnson JC. Multicenter osteopathic pneumonia study in the elderly: subgroup analysis on hospital length of stay, ventilator-dependent respiratory failure rate, and in-hospital mortality rate. J Am Osteopath Assoc 2016;116: 574-87.

9. Noll DR, Degenhardt BF, Fossum C, Hensel K. Clinical and research protocol for osteopathic manipulative treatment of elderly patients with pneumonia. J Am Osteopath Assoc 2008; 108:508-16. [Erratum in: J Am Osteopath Assoc. 2008;108(11): 670].

10. Marin T, Maxel X, Robin A, Stubbe L. Evidence-based assessment of potential therapeutic effects of adjunct osteopathic medicine for multidisciplinary care of acute and convalescent COVID-19 patients. Explore (NY) 2020. https://doi.org/10.1016/j.explore. 2020.09.006. 